\title{
ROMAIN GANDIA
}

IREGE, université de Savoie; ESC Chambéry

\section{Innovation ouverte et management de la propriété intellectuelle}

\section{Quelles stratégies dans le jeu vidéo?}

\begin{abstract}
Nombreuses sont les difficultés de maîtrise et de valorisation des droits de propriété attachés à l'innovation collaborative. La littérature indique une relation négative entre le niveau d'ouverture du processus d'innovation et la détention des droits de propriété. Toutefois, elle ne précise ni le type de collaboration ni la nature des partenaires. À travers l'étude de cinq studios de jeu vidéo, nous mettons en évidence que l'ouverture du processus d'innovation par une collaboration fermée avec des partenaires implique une relation négative entre niveau d'ouverture et détention des droits de propriété intellectuelle. À l'inverse, une collaboration ouverte sur des communautés d'utilisateurs conduit à un lien positif.
\end{abstract}


$\mathrm{D}$ e nombreuses entreprises abandonnent aujourd'hui leur modèle d'innovation fermé pour s'ouvrir vers l'extérieur afin de capter de nouvelles opportunités (Chesbrough, 2003a). L' « innovation ouverte » permet d'exploiter de façon stratégique les sources d'idées (Chesbrough, 2003a, 2003b) à travers des collaborations fermées et/ou ouvertes (Pisano et Verganti, 2008) rassemblant tous types de partenaires (concurrents, fournisseurs, utilisateurs, experts, etc.). Toutefois, les entreprises qui adoptent ce modèle d'innovation ouverte sont confrontées à un certain nombre de difficultés, notamment en matière de propriété intellectuelle (PI) et d'appropriation de la valeur qui lui est associée. Peu de recherches se sont intéressées à cette question. Cet article, qui s'inscrit donc dans le prolongement de Chesbrough (2003a, 2003b), Pisano et Teece (2007) et Pisano et Verganti (2008), a pour objectif d'explorer les stratégies de PI dans les modèles « ouverts » (Chesbrough, 2003a) en étudiant le lien entre la capacité d'une entreprise à tirer profit de sa PI et le niveau/type d'ouverture de son processus d'innovation.

Cet article est organisé de la manière suivante. Dans une première partie seront exposées les difficultés qui accompagnent la gestion de la PI dans les modèles d'innovation ouverte. La deuxième partie justifie le choix du terrain (le jeu vidéo) et présente les spécificités de la propriété intellectuelle dans ce secteur. Le jeu vidéo est particulièrement adapté à notre problématique car il combine des entreprises avec des logiques de PI épineuses ${ }^{1}$ du fait de la nature même des produits culturels. La troisième partie est dédiée à la présentation de la méthodologie et des cinq cas. Enfin, dans la dernière partie, nous discutons les résultats avant de conclure et de proposer des pistes de recherche.

\section{I - LA GESTION DE LA PROPRIÉTÉ INTELLECTUELLE : ENJEUX DANS L'INNOVATION OUVERTE}

L' « innovation ouverte » constitue un nouveau paradigme en réponse aux évolutions des contraintes et des opportunités des processus d'innovation. L'ouverture se caractérise par toutes les formes de coopérations externes avec tous types de partenaires (concurrents, fournisseurs, utilisateurs, clients, etc.) - mais sans qu'une distinction ne soit faite entre formes et types de collaboration. Pisano et Verganti (2008) mettent en évidence différentes formes de collaboration (ouverte/fermée) selon la façon dont les partenaires sont sélectionnés. Lorsque l'ouverture est orientée vers un nombre de partenaires identifiés et sélectionnés, l'ouverture est en « collaboration fermée ». Selon eux, une collaboration est « ouverte » lorsque le nombre et le type de partenaires sont indéterminés. Pour caractériser les modalités d'ouverture, la distinction entre collaboration ouverte et fermée apporte une vision plus précise des relations entre des partenaires sans toutefois aborder la problématique de la propriété intellectuelle.

1. En comparaison avec d'autres industries (automobile, pharmaceutique, etc.), les logiques de PI dans les industries culturelles sont complexes car le brevet n'existe pas pour les produits culturels. La protection se fait par le droit d'auteur et/ou d'autres droits spécifiques aux jeux vidéo dans un cadre juridique non stabilisé. 


\section{Propriété intellectuelle et modèle d'innovation ouverte}

La propriété intellectuelle (PI) se définit comme l'ensemble des droits exclusifs accordés sur les créations intellectuelles (Lévêque et Manière, 2003). Elle se subdivise en deux branches: 1) la propriété littéraire et artistique, qui s'applique aux œuvres de l'esprit (composée du droit d'auteur, du copyright et des droits voisins) et que l'on trouve dans l'industrie du logiciel ou du cinéma par exemple; 2) la propriété industrielle, qui regroupe les créations utilitaires (brevet d'invention et le certificat d'obtention végétale) et les signes distinctifs (la marque commerciale, le nom de domaine et l'appellation d'origine). Pour une entreprise, il s'agit de trouver un niveau d'ouverture lui permettant de garantir, d'une part, la détention des droits de PI et, d'autre part, de capter des opportunités pour soutenir ses activités d'innovation.

\section{Les applications empiriques sur la relation entre $P I$ et ouverture de l'innovation}

L'ouverture rend difficile la valorisation et l'exploitation de la PI car elle augmente le risque de fuites de connaissances protégées et de retombées involontaires, que ce soit au sein d'une collaboration fermée ou d'une collaboration ouverte. Des partenaires opportunistes peuvent s'approprier les fruits de cette collaboration, diminuant ainsi les bénéfices de l'entreprise initiatrice (Chesbrough et Appleyard, 2007). Cette difficulté est accrue dans le cas de relations entre partenaires de taille différente du fait d'éventuelles relations de dépendance et asymétries de pouvoir. Contrairement à un processus d'innovation fermé, un processus ouvert réduit la possibilité de valoriser/exploiter la PI. Il semble donc y avoir une relation négative entre le niveau d'ouverture et la valorisation/ exploitation de la PI.

La littérature sur l'innovation ouverte quant à elle s'est surtout intéressée aux collaborations avec des utilisateurs (ou communautés d'utilisateurs), certaines entreprises parvenant à s'approprier de la valeur en fédérant une communauté autour d'une de leurs innovations ${ }^{2}$, par l'accès en toute légitimité aux innovations créées par les utilisateurs (Jeppesen et Frederiksen, 2006). Dans ce type de collaboration, une relation positive entre le niveau d'ouverture du processus d'innovation et la valorisation/exploitation de la PI peut donc être envisagée.

L'innovation ouverte repose ainsi sur une gestion délicate du lien entre PI et ouverture du processus d'innovation. A priori négatif (selon Chesbrough), il pourrait aussi être envisagé comme positif sous certaines conditions et dans certains contextes. À ce titre, il semble intéressant d'étudier les conditions qui poussent les entreprises à choisir tel ou tel niveau d'ouverture du processus d'innovation. Le travail empirique vise à expliquer ce lien entre la valorisation de la PI et le niveau d'ouverture des entreprises dans le jeu vidéo, industrie culturelle soumise au droit d'auteur (comme toute œuvre multimédia) et à l'œuvre collective.

2. Le cas de l'Open Source, largement traité dans la littérature, est spécifique dans la mesure où la problématique de la protection ne se pose alors pas, l'innovation impliquant par essence l'ouverture. Par ailleurs, les motivations des membres de cette communauté sont spécifiques, et incluent notamment le don/contre don (Loilier et Tellier, 2004). 


\section{II - LES SPÉCIFICITÉS DE LA PI DANS L'INDUSTRIE DU JEU VIDÉO}

Notre choix s'est porté sur l'industrie du jeu vidéo pour trois raisons:

- le jeu vidéo est un mélange complexe de logiciel, d'art et d'interactivité. Il implique un régime d'innovation soutenu (forte créativité et originalité) soumis à des contraintes temporelles et concurrentielles élevées (Storz, 2008). Les problématiques d'innovation ouverte sont très présentes et les entreprises cherchent constamment à innover, seules ou avec des partenaires extérieurs et/ou des utilisateurs;

- les entreprises sont soumises à des logiques complexes de PI du fait de la nature même du jeu vidéo, dont la réalisation nécessite plusieurs éléments (personnages, décors, scénario, logiciel, interactivité, musique, etc.) pouvant être soumis à des droits différents de propriété littéraire et artistique. Aussi, le jeu vidéo bénéficie-t-il d'un statut juridique encore instable;

- l'industrie est structurée autour d'un processus d'innovation fragmenté (Storz, 2008). Cette répartition implique des phénomènes de dépendance entre les entreprises innovantes situées en amont (les fabricants de consoles détenteurs de la technologie) et les entreprises disposant des capacités d'accès au marché en aval (éditeurs et distributeurs). Elle est donc particulièrement adaptée pour étudier le lien entre la capacité d'appropriation de la valeur liée à l'exploitation de la PI et le niveau d'ouverture du processus d'innovation. On y trouve les fabricants de consoles, les prestataires, les studios, les éditeurs et les distributeurs. Les studios et les éditeurs sont les plus confrontés aux problématiques de PI.

\section{Le statut et la protection de la PI dans le jeu vidéo}

Le jeu vidéo souffre d'une absence de statut juridique clairement défini. Selon les spécialistes (juristes, avocats, etc.), ce n'est ni un logiciel ni une œuvre audiovisuelle. Face à cette complexité, la justice a décidé d'accorder un statut particulier au jeu vidéo, celui « d'œuvre collective » qui se définit par sa création, à l'initiative et sous la direction d'une personne morale (ou physique) qui va être investie des droits d'auteur, et son statut " collectif », qui implique un regroupement de contributions en vue d'une répartition collective des apports. $\mathrm{Ce}$ régime attribue les droits à une seule entité, ce qui rend difficile les opérations de codéveloppement d'un jeu. Pour palier cette limite, un autre régime a été développé en parallèle, l'œuvre de collaboration, qui est une copropriété commune des coauteurs. Le contrat entre coauteurs prévoit la nature des contributions de chacun, les modalités d'exploitation commune et les possibilités d'exploitation séparée. La question de la PI et des DPI est donc centrale dans ce type de régime (voir tableau 1).

\section{Les transactions de PI dans l'industrie du jeu vidéo}

Différents scénarios économiques sont liés aux transactions d'une PI (voir figure 1). Dans la majorité des cas, c'est le studio qui est à l'origine de la PI (cas des scénarios 1, 2 et 3). Celle-ci peut également provenir d'un éditeur si celui-ci possède un studio interne (cas des scénarios 4 et 5). Les relations contractuelles entre ces deux acteurs sont particulières. Lorsqu'un éditeur finance le développement d'un jeu créé par un studio, les deux parties souscrivent à un 


\section{PETIT APERÇU DE LA PROPRIÉTÉ INTELLECTUELLE DANS LE JEU VIDÉO}

La protection est inhérente à la création d'un jeu vidéo, considéré comme une « œuvre » multimédia. Lorsqu'il s'agit d'une œuvre collective, l'entreprise est protégée selon les droits d'auteur. Le droit d'auteur couvre l'ensemble des éléments de l'œuvre (personnages, décors, partie logiciel, etc.). Pour en faire le commerce, l'entreprise procède à une cession temporaire des droits d'exploitation. En échange, l'entreprise propriétaire obtient une rémunération basée sur les droits d'auteurs, généralement sous forme de royalties ${ }^{3}$. Lorsqu'il s'agit d'une œuvre de collaboration, les entreprises peuvent procéder de deux manières : la cession ou le partage des droits d'exploitation. Le premier cas, le plus courant, illustre une situation de prestation. Plusieurs partenaires participent au développement d'un jeu mais, au final, une seule entreprise conserve les droits. Les autres partenaires cèdent les droits d'exploitation liés à leur contribution en échange d'une rémunération basée sur les droits d'auteurs. Le deuxième cas, moins fréquent, illustre une situation dans laquelle les partenaires souhaitent conserver la partie des droits associée à leur contribution. La propriété reste commune et la rémunération est partagée en fonction des contributions de chacun.

contrat d'édition (AFJV, 2009) qui implique un financement " étape par étape » (appelé « Mylestones »). L’objectif pour l'éditeur est d'éviter un investissement en une seule fois ${ }^{4}$ : l'éditeur finance $20 \%$ du développement puis, si le développement lui convient, $20 \%$ de plus, etc. Dans le cas contraire, il peut arrêter le financement (scénarios 1 et 2). Généralement, le studio est contraint de «mettre de côté »sa liberté éditoriale pour suivre les directives de l'éditeur. Ces tensions entre la volonté de préserver la liberté éditoriale et les contraintes impliquées par le financement touchent en premier lieu les studios. C'est pourquoi nous choisissons de n'étudier ici que les scénarios 1,2 et 3 qui illus- trent ces tensions, les scénarios 4 et 5 étant assimilés davantage à des contrats de soustraitance qu'à des démarches d'ouverture.

\section{III - STRATÉGIES OUVERTES : CINQ ÉTUDES DE CAS}

La revue de la littérature empirique a permis d'identifier les scénarios liés aux transactions de PI. Afin d'étudier le processus d'innovation dans le développement de produits et les stratégies utilisées pour gérer et rester propriétaire des DPI, nous avons étudié, entre septembre 2008 et septembre 2009, cinq studios de taille différente et se situant dans un des trois scénarios retenus pour notre étude. Des

\footnotetext{
3. Dans le jeu vidéo, les royalties, royautés ou redevances sont les sommes versées au titulaire d'une PI en contrepartie de l'exploitation commerciale ou industrielle de cette PI.

4. Un jeu vidéo sur console nécessite aujourd'hui entre 150000 et 450000 euros d'investissement, ce qui représente une somme et un risque trop importants pour un studio de petite ou moyenne taille.
} 
Figure 1 - Scénarios de transactions de PI dans l'industrie du jeu vidéo ${ }^{5}$

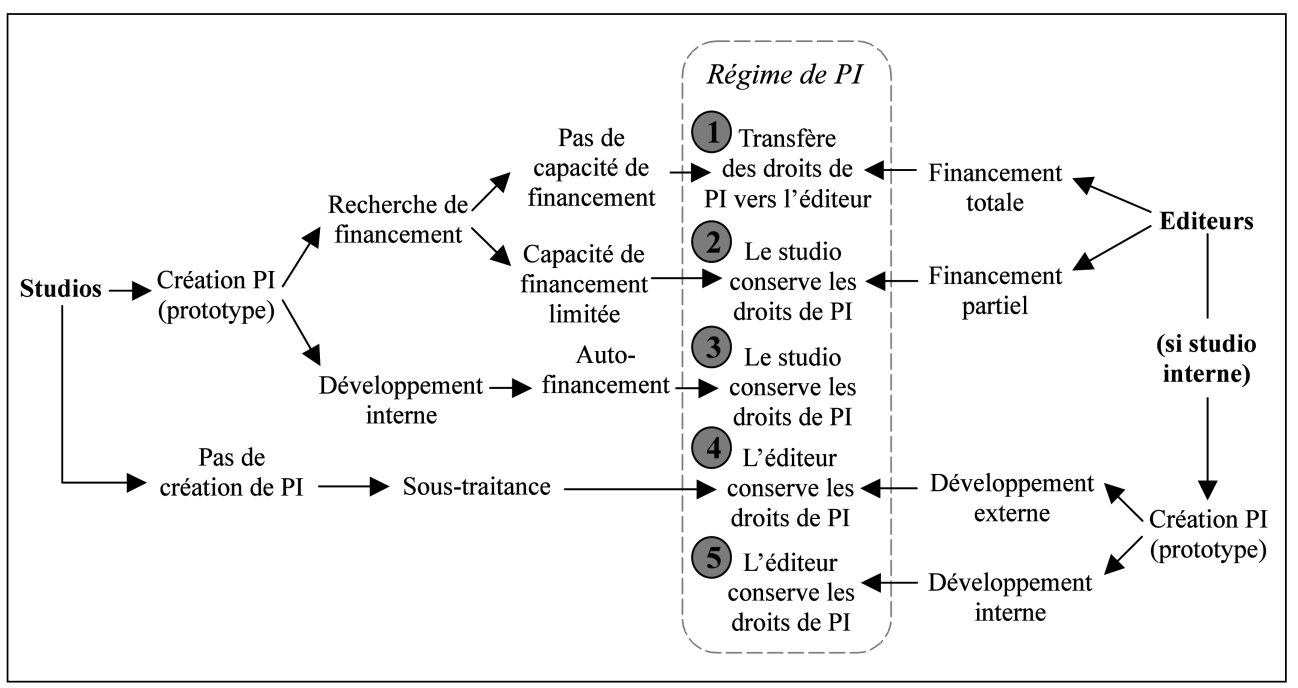

accords de confidentialité ayant été signés avec ces acteurs, nous avons utilisé des pseudonymes: la « société V 》 (petite: 17 personnes); la « société $\mathrm{W} »$ (moyenne: 34 personnes); la « société $\mathrm{X}$ » (grande: 450 personnes); la « société $\mathrm{Y}$ » (division de 80 personnes d'une grande agence de communication interactive de 450 salariés) et la " société $Z$ » (petite: 12 personnes). Notre démarche de recherche qualitative exploite des données primaires issues de 18 entretiens semi-directifs (d'une durée comprise entre $1 \mathrm{~h}$ et $1 \mathrm{~h} 30$ ) et des données secondaires issues de recherches sur internet et de journaux professionnels ${ }^{6}$.

Studio V: créé en 2003, composé de 10 personnes, il est spécialisé dans la réalisation de jeux vidéo d'aventure sur console portable. $75 \%$ de son chiffre d'affaires (CA) provient du développement de l'activité de prestation (sous-traitance) pour les éditeurs. Le reste de l'activité est constitué par des idées de jeux (il est à l'origine de la PI) sous le régime de l'œuvre collective, mais que le studio n'a pas la capacité financière de développer. Il sollicite donc des éditeurs pour obtenir des financements. Pour les convaincre, le studio conçoit des prototypes de jeu sur la base d'un document de conception et d'une vidéo de démonstration. L'éditeur exige alors la cession des DPI, le studio agissant en simple prestataire. Le contrat d'édition fait office de contrat de prestation et de contrat de cession des DPI. L'avantage pour le studio est qu'il prend peu de risque financier; en

5. Cf. littérature empirique (e.g. Le Diberder, 2002; Parmentier et Mangematin, 2009).

6. Pour des motifs de taille de l'article, nous n'avons pas inséré d'annexe méthodologique détaillant la méthode de recueil, de codage, de traitement et d'analyse des données. Cette annexe est disponible sur demande. 
revanche, il perd les droits liés à la création de ses œuvres et, si les éditeurs décident de faire une suite du jeu, le studio n'est pas en mesure de faire valoir son droit d'antériorité sur l'œuvre ${ }^{7}$. De plus, si les éditeurs stoppent la relation, ces derniers récupèrent l'ensemble des développements. Dans ce scénario 1 (figure 1), l'éditeur assume seul le risque financier et le studio perd sa liberté éditoriale. Les retours économiques sont faibles (environ $5 \%$ du prix de vente; Le Diberder, 2002).

Aujourd'hui, V exerce un autre type d'activité: la cocréation de jeux avec d'autres studios de taille moyenne qui désirent se diversifier sur les consoles mobiles. Ces derniers sollicitent $\mathrm{V}$ en qualité d'expert et l'intègrent dans leur processus de développement. V n'intervient que dans la phase de production (au niveau de la programmation de la jouabilité) et de conception du prototype (au niveau des spécificités technologiques liées aux consoles mobiles). Il conserve les DPI puisque l'éditeur n'a pas besoin de financer la totalité du jeu. Au niveau économique, la part de rétribution est calculée en fonction du niveau de participation de $\mathrm{V}$ à la cocréation du jeu. Sur les $15 \%$ des royalties attribuées par l'éditeur au studio partenaire, $V$ ne perçoit qu'entre 3 à $7 \%$ de cette somme future, en fonction de son niveau d'implication. Au final, l'ouverture semble être, pour V, une « bonne opportunité » en termes de PI puisque le régime d' « œuvre de collaboration » mis en place permet aux partenaires de partager les droits et la rémunération selon leurs contributions respectives. Cette stratégie d'ouverture (collaboration horizontale) permet au studio $\mathrm{V}$ de passer du scénario 1 au scénario 2 et d'en retirer les bénéfices artistiques (détention d'une partie des DPI, à hauteur d'environ $30 \%$ ) et financiers (augmentation jusqu'à $7 \%$ du montant total des royalties attribuées par l'éditeur).

Studio W: studio composé de 34 personnes en 2009, il a débuté en 2004 avec 23 personnes dans une activité de développement de jeux sur consoles mobiles. En 2006, la société atteint 28 personnes et commence à investir dans la création de prototypes de jeu. En 2007, elle se lance dans la création d'univers virtuels sur Internet basés sur l'exploitation de sa PI. Elle fait appel à une entreprise de l'animation spécialisée dans la production de mini-séries sur Internet ainsi qu'à une agence de communication interactive qui réalise des sites Internet. $\mathrm{W}$ a donc pour objectif de codévelopper un prototype afin d'intégrer les dimensions animation et Internet. Les deux partenaires sont intégrés dans le processus d'innovation « ouvert» de W au niveau de la conception et la préproduction. L'investissement s'est avéré minime, les produits étant diffusés en ligne ${ }^{8}$. La relation entre $\mathrm{W}$ et l'agence de communication interactive a pris la forme d'un contrat de prestation (donc cession des DPI liés à la réalisation du site interactif). Pour le studio d'animation, des droits de diffusion ont été négociés et

7. Certains studios parviennent à obtenir gain de cause grâce à une jurisprudence qui leur est souvent favorable. Toutefois, les studios ignorent généralement qu'ils disposent d'un droit sur leur création - même après cession de ces droits. Le jeu vidéo étant une œuvre multimédia, la protection est inhérente à sa création. Ce qui, au plan juridique correspond à la notion "d'œuvre composite ».

8. En principe, l'accès au client final est coûteux à cause du pouvoir des distributeurs qui verrouillent l'accès au marché. 
cédés sur le principe d'un paiement fixe pour l'ensemble des épisodes d'une minisérie. Le studio d'animation conserve donc les DPI. Pour W, cette collaboration lui a permis de valoriser une PI. Même si la rentabilité est faible compte tenu du succès mitigé du jeu (un peu plus de 1000 abonnés correspondant à $10 \%$ du CA de $\mathrm{W})$, l'entreprise peut étendre sa liberté éditoriale en proposant et diffusant ses propres contenus. La stratégie d'ouverture du studio W lui permet de passer du scénario 1 au scénario 3 dans lequel la capacité d'autofinancement est acquise grâce à la collaboration horizontale avec d'autres studios de secteurs d'activités différents.

Studio X: composé de 450 personnes en 2009, ce studio est spécialisé dans la production de jeu sur plateformes (consoles de salon, portables, téléphones mobiles, etc.). Créé en 1992 avec 14 employés, X a débuté son activité comme prestataire pour les éditeurs. Très vite, le succès lié à ses produits lui permet d'investir afin de créer ses propres jeux. En 2000, la société se lance dans la cocréation d'un jeu sur console avec un studio partenaire. Le studio X était donc dans une situation " classique » (scénario 2), avec une stratégie d'ouverture destinée à diminuer les contraintes liées au financement par l'éditeur. Au fil des années, le succès de ses produits lui a permis de développer sa capacité de financement et de s'agrandir. En 2004, avec 180 employés, il crée une œuvre et finance une grande partie de son développement pour conserver ses droits (environ $50 \%$ ) et s'assurer une plus grande liberté éditoriale. Depuis 2004, il développe et finance partiellement ses œuvres sans partenaire avec un soutien modéré de la part des éditeurs (entre 40 et $50 \%$ du budget total). Cette activité repré- sente $60 \%$ du CA de l'entreprise, le reste provenant de la sous-traitance pour le compte des éditeurs. Le studio X reste donc dans le scénario 2 mais avec un financement au moins équivalent à celui des éditeurs.

Studio Y: composé de 80 personnes en 2009, il représente la division « jeux vidéo » d'une grande agence de communication interactive (450 personnes) située à la périphérie de plusieurs industries culturelles (jeu vidéo, animation, édition, Internet et évènementiel). Créé en 2002, le studio se spécialise dans le développement et la production de jeux en ligne massivement multi-joueurs (MMORPG) - dont l'avantage est qu'il nécessite un faible coût de développement et de diffusion. Le studio dispose d'une capacité financière suffisante pour développer, produire et commercialiser ses jeux, il peut détenir l'ensemble des droits et de la valeur associés à la création. Son premier jeu connaît un succès rapide (en trois ans), ce qui lui garantit l'autonomie financière (augmentation de $30 \%$ par an en moyenne des bénéfices depuis 2005). Dès le départ, le studio est donc positionné dans le scénario 3 .

En 2007 est lancé un nouveau concept d'œuvre basé sur la création d'un univers artistique (personnages, décors), qui est décliné à travers différents médias (MMORPG, série animée TV, mangas et bandes dessinées, Web communautaire 2.0 , etc.). Y se charge du développement du MMORPG et décide d'intégrer un outil de cocréation avec les utilisateurs. Les joueurs ont la possibilité de créer et d'améliorer l'environnement du jeu. Au niveau de la PI, le studio utilise un système de protection qui lui permet de s'accaparer les droits liés aux créations des utilisateurs: le «Contrat de licence d'utilisateur 
final $(\mathrm{CLUF})^{9}$ ». Grâce à cette protection, $\mathrm{Y}$ peut ouvrir son processus d'innovation aux utilisateurs tout en conservant les droits sur sa PI et sur les créations qui lui sont associées. De ce fait, le studio reste positionné dans le scénario 3 mais accroît les bénéfices de l'innovation grâce à une stratégie d'ouverture avec les utilisateurs.

Studio Z: créé en 2000, composé de 12 personnes en 2009, il est spécialisé dans le développement et la production de jeux de sport, notamment de simulation de courses de voile sur ordinateur. La technologie utilisée pour développer le jeu étant moins coûteuse que celle d'un jeu sur console (de même que le support de diffusion utilisé), le studio peut financer l'ensemble de son développement ainsi que la commercialisation. Il conserve donc les DPI et sollicite un distributeur pour assurer le marketing et la distribution du jeu. Même si le studio prend plus de risques en assumant l'ensemble du financement lié au développement du jeu, il conserve toute sa liberté éditoriale et augmente ses retours économiques. En revanche, il doit céder une partie de la valeur au distributeur (environ $40 \%$ du prix de vente; Le Diberder, 2002). Cette situation illustre le scénario 3 mais, contrairement à $\mathrm{Y}, \mathrm{Z}$ doit ici recourir à un distributeur. En 2003, il réalise un jeu de course automobile sur ordinateur qui connaît un succès rapide (plus de 500000 exemplaires vendus en 2006). Il ouvre son processus d'innovation aux utilisateurs en intégrant un outil de création de circuits avec des contrats CLUF permettant à $Z$ d'être propriétaire des droits liés aux créations des utilisateurs. Aujourd'hui, plus de 5600000 utilisateurs sont enregistrés et plusieurs millions de circuits ont été créés. Tout comme le studio Y, Z est positionné dans le scénario 3 et diffuse son jeu sur Internet avec une stratégie d'ouverture avec les utilisateurs.

\section{IV - RÉSULTATS ET DISCUSSION}

Cette recherche montre que les acteurs innovants $\mathrm{du}$ secteur du jeu vidéo sont contraints d'articuler différents niveaux d'ouverture pour créer et protéger la valeur issue de leur activité innovante. L'ouverture devient ainsi le moyen pour se libérer du verrouillage éditorial des acteurs de l'aval (les éditeurs). Dans cette perspective, cette recherche questionne la pertinence entre les modèles « ouverts » et « fermés » (Chesbrough, 2003a) et contribue à la littérature émergente sur cette thématique. Cette même littérature postule implicitement qu'il existe une relation négative entre degré d'ouverture du processus d'innovation et détention/valorisation des droits de propriété issus de l'innovation qui en résulte. Les résultats de notre recherche montrent que cette relation n'est pas univoque. En effet, le sens de la relation entre détention et valorisation des droits liés à l'innovation et niveau d'ouverture varie selon que l'ouverture du processus repose sur une collaboration fermée ou ouverte (Pisano et Verganti, 2008). Dans les cas étudiés, la collaboration fermée concerne des

9. Ce contrat autorise l'entreprise à devenir propriétaire des droits liés à la création d'un joueur. Lorsqu'un joueur crée un objet, un scénario, etc., il doit souscrire au CLUF. Celui-ci prend généralement la forme d'une fenêtre où le joueur doit cocher « j'accepte » pour permettre à sa création d'exister dans le jeu. S'il refuse, sa création est supprimée. Le joueur n'a donc pas vraiment le choix, il doit céder ses droits pour créer. 
relations entre les entreprises du secteur et la collaboration ouverte repose sur des communautés d'utilisateurs.

\section{DPI et niveau d'ouverture : les collaborations fermées}

Un studio qui crée une œuvre culturelle en interne (innovation « fermée », Chesbrough, 2003b) et détient les droits liés à cette œuvre, peut néanmoins ne pas pouvoir être en mesure de s'approprier la valeur associée à ces droits ( $\mathrm{V}$ et $\mathrm{X}$ à leurs débuts scénario 1). On rejoint ici la problématique soulevée par Pisano et Teece (2007): les actifs complémentaires (édition, distribution) n'étant pas possédés par les studios, ces derniers se trouvent contraints de céder leurs rentes (les DPI) aux éditeurs pour donner vie à leur innovation. Les studios se trouvent dans une dépendance des ressources envers les éditeurs, qui imposent leurs choix, limitant leur liberté créative.

Pour accroître leur liberté éditoriale et leur notoriété, certains studios, même petits, n'hésitent pas à partager leur savoir-faire en s'alliant avec d'autres studios. Cette innovation « quasi-ouverte » permet à $\mathrm{V}, \mathrm{W}$ et $\mathrm{X}$ de récupérer une partie de la valeur associée à la création de leurs œuvres. Les difficultés rencontrées lors de la mise en place de tels accords (cas de V et X) semblent liées à la position de ces acteurs dans la filière (voir tableau 4 ci-après). Même si la collaboration entraîne des tensions et des conflits, notamment au niveau de la répartition de la valeur, les studios $\mathrm{V}, \mathrm{W}$ et $\mathrm{X}$ parviennent finalement à augmenter leur liberté éditoriale et à conserver leurs DPI (de manière individuelle ou partagée). Plus le studio a la capacité financière de maîtriser l'intégralité du processus d'innovation (création, développement, commer- cialisation et diffusion) et notamment le développement, moins le studio est dépendant de l'éditeur. Si le studio arrive à prendre en charge une partie du développement (scénario 2) ou à coopérer avec d'autres studios (V et $\mathrm{X}$ ), alors il peut conserver les DPI (partagés ou non) - et ne pas les céder à l'éditeur. On retrouve ici le problème de la répartition de la valeur au sein d'alliances: lorsque la répartition n'est pas négociée en amont (V et $\mathrm{X}$ ), ce qui est souvent difficile dans les contextes de collaboration complexes, les conflits sont quasiment inévitables.

Lorsqu'il aboutit, ce type d'ouverture permet donc au studio de récupérer une partie des droits d'exploitations associés à ses propres produits (selon les accords avec l'autre studio). En outre, d'autres actifs sont nécessaires pour permettre à ces studios d'atteindre les consommateurs finaux, comme les ressources pour la commercialisation et la distribution - qui ne peuvent être intégrées par des petits studios pour des raisons de taille et de ressources.

Pour V, W et X, l'ouverture leur a permis, in fine, de conserver les DPI (de manière individuelle ou partagée) et de gagner de la liberté créative (éditoriale) - et peut-être également de se construire une réputation dans la filière. La capacité d'appropriation des bénéfices (au travers des DPI) dépend largement des ressources maîtrisées par l'entreprise (voir tableau 4) - le montant de ces rentes étant toutefois conditionné par le succès commercial du jeu. Pour V, W et X, la taille et les ressources dont dispose l'entreprise jouent un rôle clé lors des négociations du pourcentage sur les ventes, ce type d'ouverture du processus d'innovation (« collaboration fermée ») étant une stratégie contrainte pour les petits studios. À l'in- 
Tableau 1 - Synthèse des 5 cas étudiés

\begin{tabular}{|c|c|c|c|c|c|c|c|}
\hline & Taille & $\begin{array}{l}\text { Position } \\
\text { dans } \\
\text { la filière }\end{array}$ & $\begin{array}{c}\text { Maîtrise } \\
\text { des ressources } \\
\text { et compétences } \\
\text { complémentaires }\end{array}$ & $\begin{array}{c}\text { Phase } \\
\text { ouverte } \\
\text { du processus } \\
\text { d'innovation }\end{array}$ & $\begin{array}{l}\text { Appropriation } \\
\text { de la valeur } \\
\text { créée } \\
\text { (DPI) }\end{array}$ & $\begin{array}{c}\text { Type de } \\
\text { collaboration }\end{array}$ & $\begin{array}{l}\text { Évolution et bénéfices } \\
\text { pour l'entreprise }\end{array}$ \\
\hline $\begin{array}{c}\text { Entreprise } \\
\mathrm{V}\end{array}$ & $\begin{array}{l}\text { Très } \\
\text { petite } \\
(10)\end{array}$ & $\begin{array}{l}\text { Studio } \\
\text { dépendant } \\
\text { d'un ou } \\
\text { plusieurs } \\
\text { éditeurs }\end{array}$ & Très Faible & $\begin{array}{l}\text { Phase de } \\
\text { production }\end{array}$ & $\begin{array}{l}\text { Très faible, } \\
\text { voire } \\
\text { nulle }\end{array}$ & $\begin{array}{l}\text { Collaboration } \\
\text { fermée } \\
\text { Inter- } \\
\text { organisationnelle }\end{array}$ & $\begin{array}{c}\text { Passage du scénario } 1 \\
\text { au scénario } 2 \\
\text { (accroissement } \\
\text { des bénéfices liés } \\
\text { au partage des DPI) }\end{array}$ \\
\hline $\begin{array}{c}\text { Entreprise } \\
\text { W }\end{array}$ & $\begin{array}{l}\text { Petite } \\
\text { (34) }\end{array}$ & $\begin{array}{c}\text { Studio } \\
\text { partiellement } \\
\text { dépendant }\end{array}$ & Faible & $\begin{array}{c}\text { Phases } \\
\text { de conception } \\
\text { et préproduction }\end{array}$ & Faible & $\begin{array}{l}\text { Collaboration } \\
\text { fermée } \\
\text { Inter- } \\
\text { organisationnelle }\end{array}$ & $\begin{array}{c}\text { Passage du scénario } 1 \text { au } \\
\text { scénario } 3 \text { (accroissement } \\
\text { de la capacité de } \\
\text { financement) }\end{array}$ \\
\hline $\begin{array}{c}\text { Entreprise } \\
\mathrm{X}\end{array}$ & $\begin{array}{l}\text { Grande } \\
(450)\end{array}$ & $\begin{array}{l}\text { Studio } \\
\text { partiellement } \\
\text { dépendant }\end{array}$ & Modérée & $\begin{array}{l}\text { Phases } \\
\text { de conception } \\
\text { et de } \\
\text { préproduction }\end{array}$ & Modérée & $\begin{array}{l}\text { Collaboration } \\
\text { fermée } \\
\text { Inter- } \\
\text { organisationnelle }\end{array}$ & $\begin{array}{c}\text { Scénario } 2 \\
\text { (augmentation } \\
\text { de la capacité } \\
\text { de financement) }\end{array}$ \\
\hline $\begin{array}{l}\text { Division Y } \\
\text { d'une } \\
\text { agence de } \\
450 \text { pers. }\end{array}$ & $\begin{array}{c}\text { Moyenne } \\
(80)\end{array}$ & $\begin{array}{l}\text { Studio éditeur } \\
\text { distributeur } \\
\text { indépendant }\end{array}$ & Forte & $\begin{array}{l}\text { Phase de } \\
\text { conception }\end{array}$ & Forte & $\begin{array}{c}\text { Collaboration } \\
\text { ouverte } \\
\text { Communauté } \\
\text { d'utilisateurs }\end{array}$ & $\begin{array}{c}\text { Scénario } 3 \\
\text { (accroissement } \\
\text { des bénéfices } \\
\text { de l'innovation) }\end{array}$ \\
\hline $\begin{array}{c}\text { Entreprise } \\
\quad \mathrm{Z}\end{array}$ & $\begin{array}{l}\text { Très } \\
\text { petite } \\
(12)\end{array}$ & $\begin{array}{c}\text { Studio éditeur } \\
\text { partiellement } \\
\text { dépendant }\end{array}$ & $\begin{array}{l}\text { Modérée (dépendance } \\
\text { initiale envers les } \\
\text { distributeurs) puis } \\
\text { Forte (depuis } \\
\text { Internet) }\end{array}$ & $\begin{array}{l}\text { Phase de } \\
\text { conception }\end{array}$ & Forte & $\begin{array}{c}\text { Collaboration } \\
\text { ouverte } \\
\text { Communauté } \\
\text { d'utilisateurs }\end{array}$ & $\begin{array}{c}\text { Scénario } 3 \\
\text { (accroissement des } \\
\text { bénéfices de l'innovation } \\
\text { et diminution des coûts } \\
\text { de distribution) }\end{array}$ \\
\hline
\end{tabular}


verse, les grands studios, qui disposent d'une capacité financière suffisante pour assumer seuls la création et le développement de leur PI (X aujourd'hui), n'ont pas/plus intérêt à ouvrir leur processus d'innovation. Ici, l'ouverture n'apparaît que comme une stratégie temporaire, un risque à prendre pour sortir d'une situation de dépendance trop contraignante - et non une stratégie volontaire de dynamisation de l'innovation (Chesbrough, 2003b).

Nos cas d'ouverture par collaboration fermée mettent en évidence une relation néga- tive entre ces deux dimensions. Le caractère temporaire de l'ouverture montre ici que les studios de petite taille, aux ressources limitées, prennent un risque pour capturer de nouveaux actifs afin de conquérir leur autonomie financière et éditoriale. En cas de succès, elles ont intérêt à refermer leur processus d'innovation pour maîtriser les DPI associés à leurs créations. Dans le cas de X, l'alternance entre ouverture et fermeture décrit ainsi une courbe en U inversée entre niveau d'ouverture et détention/valorisation des droits de PI (cas $\mathrm{X}$ - figure 2).

Figure 2 - Niveau d'ouverture et valeur tirée de la PI

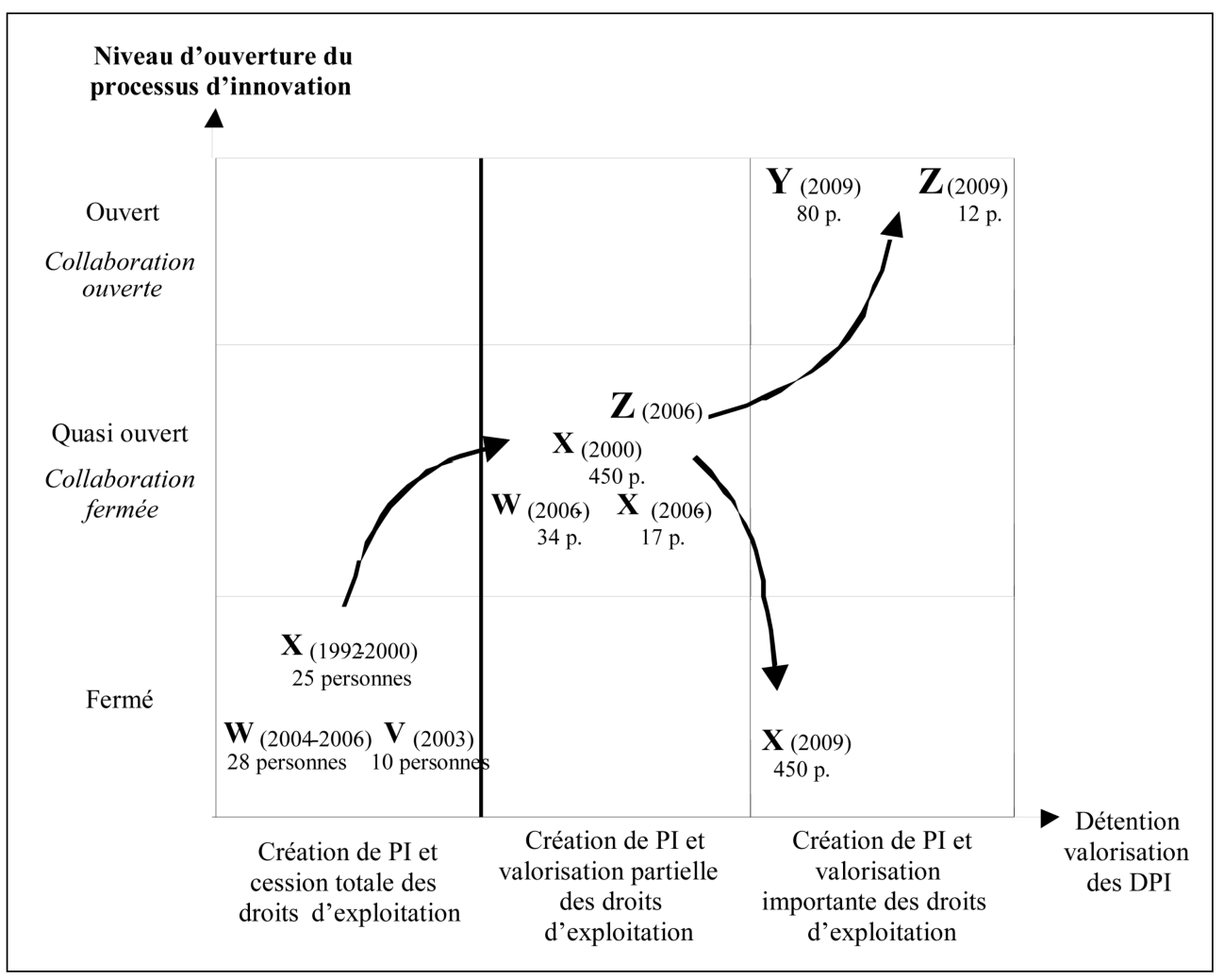

10. Il s'agit de la motivation intrinsèque que l'on trouve notamment dans l'Open Source, tels le plaisir de créer, la recherche de réputation, la réciprocité, le respect des pairs. 


\section{DPI et niveau d'ouverture : les collaborations avec les communautés d'utilisateurs}

La collaboration ouverte se distingue fortement du premier type. En effet, pour Y et Z, il ne s'agit pas de sélectionner un partenaire doté de ressources, supplémentaires (cas V et X) ou complémentaires (cas W), mais plutôt d'utiliser directement le potentiel créatif des utilisateurs finaux du jeu. Cette stratégie d'ouverture requiert un accès à ces derniers. Plutôt que d'utiliser les canaux de distribution classiques, les studios $\mathrm{Y}$ et $\mathrm{Z}$ ont utilisé Internet - sans pour autant disposer de ressources financières importantes (pour $\mathrm{Z}$, suite au succès du jeu sur PC, pour Y dès le départ). La valeur ainsi créée par (et pour) les utilisateurs s'accroît avec la taille de la communauté. Ici, l'ouverture du processus d'innovation n'est pas antinomique avec la conservation des DPI, conservés en totalité. Ceci s'explique par le fait que, pour l'instant, la motivation intrinsèque ${ }^{10}$ des joueurs est forte et ne les incite pas à avoir d'autres revendications. L'attrait pour les nouveautés entretient l'assiduité et la motivation de la communauté, générant un cercle vertueux qui, au moyen du CLUF, permet au studio de bénéficier d'une dynamique créative auto-entretenue qui valorise son produit. Nous prolongeons les résultats sur les modes de collaboration pour innover (Pisano et Verganti, 2008) en mettant en relation le niveau d'ouverture du processus d'innovation des studios (cas Y et Z) avec leur capacité à valoriser leur propriété intellectuelle (cf. figure 2).

La collaboration ouverte de $\mathrm{Y}$ et $\mathrm{Z}$ est, sur ce point, très différente de celle identifiée pour les autres studios (V, W et X). La figure 2 montre des trajectoires opposées entre les deux types de collaborations. Toutefois, il est important de noter que les conditions préalables à l'ouverture pour $\mathrm{Y}$ et $Z$ reposent sur la maîtrise des ressources nécessaires à l'élaboration et à la diffusion du jeu. Dans les deux cas, l'entreprise met à disposition des utilisateurs un ensemble de ressources technologiques au sein desquelles peut s'exprimer leur liberté créative. Internet permet ici clairement de se passer des acteurs " incontournables » (éditeurs, distributeurs) - et généralement bloquants (Pisano et Teece, 2007), pour accéder au marché. Ces deux dimensions (ressources et compétences, accès direct au marché sans distributeur) semblent nécessaires pour mettre en œuvre une telle stratégie d'ouverture et être en mesure d'en tirer pleinement profit. Ici, contrairement à la collaboration fermée interorganisationnelle, la taille ne semble pas être une limite à la collaboration ouverte et à son succès. En outre, dans une telle configuration de collaboration, on observe une relation positive entre niveau d'ouverture et capacité à détenir/valoriser les DPI issus des innovations. Le fait que cette relation évolue en sens inverse de celle observée au sein des collaborations fermées interorganisationnelles nous conduit à envisager que le type de collaboration joue un rôle clé sur la relation entre ouverture et détention/valorisation des DPI.

Sur la base des résultats obtenus, deux propositions peuvent être avancées:

P1. Lorsque l'ouverture du processus d'innovation repose sur une collaboration fermée interorganisationnelle, plus le niveau d'ouverture est grand, plus le potentiel de valorisation des DPI est faible. 
P2. Lorsque l'ouverture du processus d'innovation repose sur une collaboration ouverte sur les communautés d'utilisateurs, plus le niveau d'ouverture est grand, plus le potentiel de valorisation des DPI est élevé.

\section{CONCLUSION}

$\mathrm{Au}$ plan théorique, cette recherche permet de mettre en évidence qu'il existe une relation entre le niveau d'ouverture du processus d'innovation et la valorisation des droits de propriété. Dans le prolongement des travaux de Pisano et Verganti (2008), nous avons identifié que le type de collaboration (fermé vs ouvert) joue un rôle clé pour la détention et l'exploitation des droits issus de l'innovation. Par ailleurs, notre recherche fournit un éclairage intéressant sur la possibilité pour les petites entreprises dans l'industrie culturelle d'utiliser avec profit une stratégie d' " open innovation ». En effet, jusqu'à présent, les travaux se sont essentiellement focalisés sur les grandes entreprises, et sur les secteurs de la haute technologie ou l'open source.

Au plan managérial, cette recherche montre qu'il est possible d'envisager des scénarios de développement favorables indépendamment des contraintes généralement mises en avant comme la taille de l'entreprise - et donc ses ressources financières. Nous avons vu que des alternatives existent et semblent accessibles, même pour les studios de très petite taille. Toutefois, compte tenu de la diversité des studios et de leurs trajectoires de développement parfois atypiques d'une part, de la méthodologie que nous avons retenue d'autre part, nous ne pouvons prétendre à la généralisation de nos résultats. Les studios $\mathrm{V}, \mathrm{W}$ et $\mathrm{X}$ semblent ainsi représentatifs du secteur des jeux vidéo; en revanche, $\mathrm{Y}$ et $\mathrm{Z}$, qui ouvrent leur processus d'innovation aux communautés d'utilisateurs sont assez atypiques de par leurs choix, la qualité de leurs jeux - et leur succès. Il serait utile de compléter notre étude par des études empiriques plus larges et dans d'autres secteurs proches pour vérifier la solidité de nos résultats. En effet, le caractère spécifique de l'industrie étudiée ici limite la portée de nos résultats. Les stratégies de collaboration ouvertes observées pourraient-elles trouver leurs équivalents dans des industries plus « traditionnelles »?

\section{BibliograPhiE}

AFJV, « Le financement du Jeu Vidéo en France. États des lieux et perspectives », Études et conseils de l'Association Française du Jeu Vidéo, Paris, 2009.

Chesbrough H., "The Era of Open Innovation”, Sloan Management Review, vol. 44, $\mathrm{n}^{\circ} 3$, 2003a, p. 35-41.

Chesbrough H., Open Innovation: The New Imperative for Creating and Profiting from Technology, MA, Harvard Business School Press, Boston, 2003b.

Chesbrough H., "The Logic of Open Innovation: Managing Intellectual Property", California Management Review, vol. 45, $\mathrm{n}^{\circ}$ 3, 2003c, p. 33-58. 
Chesbrough H. et Appleyard M., "Open Innovation and Strategy", California Management Review, vol. 50, $\mathrm{n}^{\circ} 1,2007$, p. 57-76.

Jeppesen L. et Frederiksen L., "Why do Users Contribute to Firm-Hosted User Communities? The Case of Computer-Controlled Music Instruments", Organization Science, vol. 17, $\mathrm{n}^{\circ}$ 1, 2006, p. 45-63.

Le Diberder A., « La création de jeux vidéo en France en 2001 », Étude du ministère de la Culture et de la Communication, Paris, 2002.

Loilier T. et Tellier A., "Comment peut-on se faire confiance sans se voir? Le cas du développement des logiciels libres »,M@n@gement, vol. 7, n³, 2004, p. 275-306.

Parmentier G. et Mangematin V., « Innovation et création dans le jeu vidéo. Comment concilier exploration et exploitation? », Revue française de gestion, vol. 35, n 191, 2009, p. 77-87.

Pisano G.P. et Teece D.F., "How to Capture Value from Innovation: Shaping Intellectual Property and Industry Architecture", California Management Review, vol. 50, $\mathrm{n}^{\circ}$ 1, 2007, p. 278-296.

Pisano, G.P. et Verganti R., "Which Kind of Collaboration is Right for You?", Harvard Business Review, vol. 86, $\mathrm{n}^{\circ}$ 12, 2008, p. 79-86.

Storz C., "Dynamics in Innovation Systems: Evidence from Japan's Game Software Industry", Research Policy, vol. 37, n 9, 2008, p. 1480-1491. 
